\title{
Tecnología como impulsora del desarrollo sostenible: una mirada desde la investigación
}

\author{
Alfredo Estrada \\ aestrada@ulima.edu.pe / Universidad de Lima, Perú
}

Recepción: 24/1/2021 Aceptación: 15/2/2021

REsumen. Tomando como punto de partida el contexto actual y reflexionando sobre la manera en que se debe construir la nueva normalidad de la que se hace mención actualmente, el presente artículo pone a consideración los principales hitos en los que debe basarse esta construcción, que debe ser de carácter transformador. En ese sentido, se identifica a la tecnología como una de las contribuciones más importantes para el impulso hacia el desarrollo sostenible, se establece preliminarmente la relación "desarrollo tecnológico-desarrollo sostenible"; esto último es producto de una revisión documentaria basada en la agenda 2030, en estudios académicos y científicos sobre tecnología, inteligencia artificial y su relación con los objetivos de desarrollo sostenible. Como resultado, se resalta el rol de la academia como motor de los avances científicos para el desarrollo tecnológico y la sostenibilidad. Finalmente, se presentan insumos potenciales de investigación en campos como tecnologías responsables, desarrollo sostenible basado en la tecnología, entre otros. Lo que se pone en evidencia es la necesidad de investigar la sostenibilidad como un sistema compuesto de tres dimensiones interdependientes: una dimensión natural, una dimensión humana y una dimensión tecnológica.

PALABRAS CLAVE: desarrollo sostenible / agenda global / desarrollo tecnológico

/ inteligencia artificial / investigación / academia 


\section{Technology as a Driver of Sustainable Development: A Research Perspective}

AвSTRACT. Taking the current context as a starting point and reflecting on how to build a new normality, this article considers the main milestones on which the building of this new normality, which must be of a transforming nature, must be based. In this sense, technology is identified as one of the most important contributions to boost sustainable development, and the relationship "technological development-sustainable development" is preliminarily established as the product of a documentary review based on the 2030 Agenda for Sustainable Development, as well as on academic and scientific studies on technology, artificial intelligence and its relationship with the Sustainable Development Goals. As a result, the role of academia as an engine of scientific development for the technological development for sustainability is highlighted. Finally, potential research inputs are presented in fields such as responsible technologies, technology-based sustainable development, among others. What becomes evident is the need to investigate sustainability as a system composed of three interdependent dimensions: a natural dimension, a human dimension and a technological dimension.

KEYWORDS: sustainable development / global agenda / technological development / artificial intelligence / research / academia 


\section{INTRODUCCIÓN}

Sabemos que desde finales del 2019 e inicios del 2021 los sistemas educativos, económicos, sociales y productivos mundiales están pasando por una prueba sin precedentes; la crisis sanitaria generada por el COVID-19 nos exige preguntarnos cómo construimos realmente una "nueva normalidad" que permita la continuidad del desarrollo global.

El desarrollo de una economía basada en una demanda universal de servicios sociales básicos que reduzcan brechas y mitiguen el cambio climático, así como la construcción de un mundo donde los negocios y las organizaciones sean éticas y generadoras de externalidades positivas, son temas tan urgentes como importantes. La tecnología es un elemento que aporta al desarrollo del nuevo modus vivendi que enfrentamos, permitiendo construir posibles soluciones en beneficio de la comunidad. La intervención de la tecnología en el desarrollo sostenible es un desafío que insta a diversos actores a participar activamente. La finalidad de este documento es establecer la importancia de la investigación sobre el desarrollo tecnológico para alcanzar el desarrollo sostenible, así como poner de relieve reflexiones y aspectos de vinculación importantes para que esta área de investigación esté a la vanguardia del contexto actual en el que vivimos. Para tal efecto, se ha desarrollado una profunda revisión documentaria en bases de datos cerradas y en informes técnicos institucionales que nos permiten concluir los niveles de importancia y las vinculaciones actuales y futuras que se darán sobre este tema.

El rol de la agenda 2030, conocida como la hoja de ruta que mide los avances y ajustes que se dan a diversos ejes de acción para el desarrollo sostenible, cuenta actualmente con avances importantes, pero no suficientes; la crisis generada por la pandemia ha afectado a todos los sectores de la población a nivel mundial. Desde niños que no asisten a clases hasta problemas económicos en todas las regiones. Estos inconvenientes nos llevan a resaltar, por ejemplo, la importancia de la gestión de datos y estadísticas de forma oportuna, el aseguramiento de la calidad de datos, abiertos y desglosados, entre otras. Estas necesidades nunca han sido tan relevantes como lo son ahora, en plena crisis del COVID-19, y constituyen un claro ejemplo de cómo la tecnología impacta en el desarrollo sostenible. Sin embargo, no es lo único.

El cumplimiento de las metas de la agenda 2030 sigue siendo desigual, 230 de ellas tienen un avance importante, pero no alineado a la "nueva construcción de la normalidad"; sobre esto último es importante afirmar que no nos enfrentamos a una nueva normalidad per se, afrontamos el reto de una nueva construcción de la normalidad, de la que todos somos parte; por esta razón, debemos contar con diversos mecanismos, entre ellos la tecnología.

En ese sentido, falta un cambio transformador desde diferentes vectores, la comunidad, la industria, las leyes, la economía, entre otros, los cuales deben converger para crear un camino de respuestas a la agenda global. Al respecto, Zhenmin (2020) afirma que los actores públicos y privados de toda la comunidad global deben tener en cuenta las lecciones que se están aprendiendo en esta situación de alerta para formular un mundo sano, equitativo, resiliente y 
sostenible. La resiliencia tiene en la tecnología un aliado importante de trabajo que conduce cada vez más efectiva y eficientemente al desarrollo sostenible.

La necesidad de que la agenda global sea transformadora no es un tema nuevo; antes de la crisis generada por el COVID-19 ya se hacía necesario el diseño de estrategias más efectivas. A un año, aproximadamente, del inicio de la pandemia, se ponen cada vez más de relieve diversas carencias a nivel global, por referir algunas: un rápido deterioro del medioambiente, las vulnerabilidad de las poblaciones más pobres, las cadenas globales de alimentos profundamente impactadas, la educación y el acceso a ella cada vez más limitado, el restringido acceso a diversos recursos, el incremento del hambre, los sistemas de salud limitados, entre otros. Estos son temas de impacto que se enfrentarán en estos últimos diez años de agenda; la conversión global para el nuevo desarrollo sostenible necesita de impulsores estructurales; por ejemplo, el de la educación, tal como lo indican González-Salamanca et al. (2020); el uso de la tecnología como recurso, herramienta y andamiaje lo convierte en un factor de desarrollo de habilidades y conocimientos que se enfrentará de forma exitosa al reto de lograr el futuro sostenible que se busca desde lo laboral pasando por lo técnico y profesional. La tecnología impacta en el desarrollo de una ciudadanía global y en el desarrollo sostenible en su totalidad.

Una agenda global verdaderamente transformadora educa, forma, capacita; en ese sentido, el rol de la academia es determinante para identificar soluciones colaborativas tanto para dicha agenda como para las diversas crisis que se generan. En estos escenarios inciertos es donde se requiere información estratégica y acceso a ella, nuevos big data, modelos de negocio, financiamiento inclusivo mediante las plataformas digitales, el desarrollo de nuevos modelos de realidad, tal como señala la Asociación 2030Vision (2017). Las oportunidades y desafíos para que la tecnología avance y contribuya a los objetivos de desarrollo sostenible impactarán de manera representativa en la economía mundial; dicho esto, la influencia del desarrollo tecnológico en el desarrollo sostenible es relevante y el eje "desarrollo tecnológico-academia" constituye un impulsor estratégico para su dimensionamiento e impacto.

La educación y el desarrollo de la innovación e investigación configurada como un proceso estratégico de impacto acerca más la reflexión anteriormente citada a la línea de pensamiento que destaca CEPAL (2020) cuando indica que el sector académico desempeña una labor investigadora y docente imprescindible para progresar en la agenda 2030, indicando además que este proceso estratégico descansa en diferentes hitos tales como la investigación, la innovación y la inclusión. Así, la academia y su labor investigadora orientada a la innovación, específicamente en desarrollo y tecnología, constituye un agente de cambio para la agenda y para los futuros indicadores globales que se formulen sobre desarrollo sostenible.

La investigación es un puente importante para revisar la vinculación entre la tecnología y el desarrollo sostenible. Esta actividad impulsa el desarrollo tecnológico para el desarrollo sostenible desde un enfoque empírico y de evidencia en la empresa y la comunidad. Estos dos ámbitos son determinantes para fortalecer las líneas de trabajo de la investigación. El futuro 
del estudio del nuevo constructo "tecnología-sostenibilidad" se basa en la nueva formulación de los sistemas de gestión del conocimiento, con un abordaje tanto empresarial como comunitario. En este sentido, es bastante interesante la posición de Johannessen (1999) que sienta las bases para analizar estos sistemas como una suerte de "centros" que promueven la gestión del trabajo inteligente mediante la sinergia de factores humanos, organizacionales y tecnológicos; el intercambio de conocimiento generado produce valor a la empresa y, por ende, a la sociedad. Este aspecto de la creación de valor "empresa-sociedad" es clave porque coadyuva al establecimiento de una cultura corporativa en la compañía para explotar la tecnología en la mejora de procesos y modelos empresariales. Tal como lo indica Hoeschl (2006), el punto de partida del desarrollo sostenible es la cultura; así, la construcción cultural empresarial hacia la sostenibilidad se sustenta en la mezcla de factores humanos (individuales y sociales), factores organizacionales (colaborativos estratégicos) y factores tecnológicos (funcionales e innovadores). Dicho esto, se pone a la luz diversos ámbitos que deben trabajarse en la investigación para el desarrollo tecnológico-desarrollo sostenible, desde una lógica tridimensional; el hombre, la empresa y la tecnología son los vértices que acompañarán a esta nueva comunidad del conocimiento y la decantará en una comunidad sostenible.

En la línea de lo antes mencionado está el trabajo de Duan (2019) que sostiene que integrar la tecnología con los procesos empresariales y con diversos tipos de toma de decisiones influye en el logro de los objetivos de desarrollo sostenible, los cuales impulsan el crecimiento de la economía y el desarrollo de la comunidad. El constructo "tecnologías responsables" aparece como un espacio de oportunidad en la creación de nuevas investigaciones. Investigar las tecnologías responsables es determinante, pero también lo es el poder determinar cómo la investigación del desarrollo tecnológico en el desarrollo sostenible permite construir empresas con modelos de gestión y de inversión responsable, dado que estos dos aspectos, la gestión y la inversión, impactarán en el beneficio de la comunidad. Y esto se puede extrapolar no solo a las empresas, sino también a todo el desarrollo organizacional e institucional; no puede haber una sociedad o comunidad "sana" donde existan empresas u organizaciones que tengan insania social; la innovación tecnológica y su investigación estratégica constante permiten implementar modelos sostenibles de producción, de consumo y colaboración.

Se ha demostrado que el nivel de interacción entre los sistemas humanos, tecnológicos y naturales son impulsores de los objetivos de desarrollo sostenible. Nilsson (2016) sostiene fervientemente esta posición; se pone en evidencia la necesidad de investigar cultura sostenible basada en tecnología, los hitos que definan la nueva forma de estructurar los sistemas de gestión del conocimiento, los componentes del nuevo valor compartido basado en tecnología y los modelos de negocio sostenibles en digitalización y desarrollo tecnológico.

La investigación sobre el desarrollo tecnológico para el desarrollo sostenible, si bien data desde hace muchos años, tiene una característica muy importante que la diferencia de otros ámbitos de la investigación, su nivel de impredictibilidad es prevalente; en ese sentido, Kaplan 
(2010) sostiene que la investigación en este ámbito requiere constantemente de una comprensión y análisis dinámico que solo se logrará en investigaciones aplicadas que generen evidencia como resultado de la interacción de algunos hitos importantes del desarrollo sostenible, tales como el empleo, la ética, la educación y los negocios. Es claro que Kaplan se adelantó un poco a su tiempo, dado que estos elementos que citaba en su producción científica hoy son claves para la continuidad de la humanidad; realizar investigación sobre desarrollo tecnológico para alcanzar el desarrollo sostenible supone entender la evolución de la tecnología como una oportunidad de innovación social, empresarial e institucional.

Las bases de la actividad científica, específicamente en los ámbitos de la tecnología para el desarrollo sostenible, constituyen un aspecto importante y generador de una agenda que compromete a la academia como principal actor de procesos de investigación e innovación, y a la empresa también, dado que es un agente clave para el crecimiento económico. En este último aspecto es que se pone a la luz una tendencia de investigación importante para las empresas llamadas "con propósito", un concepto que viene incrementando trabajos de investigación y que trasladan a un ámbito del management el concepto de valor compartido, que debe ser el ADN de las organizaciones, empresas e instituciones. Es importante destacar como oportunidad de investigación el análisis de cómo los fenómenos organizacionales que son parte de una gestión con propósito vinculan también el elemento "tecnología". En este orden de ideas, los sistemas de gestión del conocimiento y desarrollo tecnológico impactan directamente en la formación de modelos de gestión sostenible, haciendo necesaria la investigación de los factores actuales que intervienen en la generación del valor compartido en el ámbito tecnológico.

La investigación en desarrollo tecnológico para el desarrollo sostenible debe fortalecer los nuevos espacios de oportunidad que el efecto del COVID-19 ha generado y que mediante la innovación se pueden mejorar. Así, la política, la capacidad de renovación, la vigilancia, el crecimiento laboral, la seguridad y la salud son espacios que la pandemia ha desconfigurado para luego volverse a configurar; la oportunidad es clara y evidente, se requieren nuevas líneas y sublíneas de investigación al respecto. Un aporte interesante es el de Di Vaio (2020) que trabaja una nueva taxonomía aplicativa para modelos de gestión sostenible y tecnología, construyendo una escalabilidad importante de los componentes graduales que se presentan en el nuevo diseño investigativo que requiere analizar el desarrollo sostenible, desde el desarrollo tecnológico.

Finalmente, hay que destacar que diversos investigadores postulan posiciones y restricciones relacionadas con los alcances de este ámbito de la investigación, básicamente por las variables éticas y de legalidad, las cuales son muy discutibles por diversas razones; dicho esto, se identifica otra "ventana de oportunidad" importante para investigar y generar evidencia de cómo la gobernanza y gestión pública, los sistemas micro y macrovigilantes, la urbanidad, los modelos ergonómicos de los sistemas inteligentes organizacionales y la gestión ética se podrían convertir en líneas de investigación y acciones claves para la agenda global vigente y por construir, esa es la característica más importante de la tecnología: es tan transformadora como cambiante. 


\section{REFERENCIAS}

Asociación 2030Vision. (2017). 2030 Vision Global Goals Technology Forum. Editorial SustainAbility.

CEPAL. (2019). La Agenda 2030 y los objetivos de desarrollo sostenible: una oportunidad para América Latina y el Caribe. Objetivos, metas e indicadores mundiales. https:// repositorio.cepal.org/bitstream/handle/11362/40155/24/S1801141_es.pdf

Di Vaio, A., Palladino, R. Hassan, R., y Escobar, O. (2020). Artificial Intelligence and Business Models in the Sustainable Development Goals Perspective: A Systematic Literature Review. Journal of Business Research, 121, 283-314.

Duan, Y. , Edwards J., y Kumar, Y. (2019). Artificial intelligence for Decision Making in the Era of Big Data-Evolution, Challenges and Research Agenda. International Journal of Information Management, 48, 63-71. https://doi.org/10.1016/ j.ijinfomgt .2019 .01 .021

González-Salamanca, J. C., Agudelo, O., y Salinas, J. (2020). Key Competences, Education for Sustainable Development and Strategies for the Development of 21st Century Skills. A Systematic Literature Review. Sustainability, 12(24). https://doi.org/10.3390/ su 122410366

Hoeschl, H. C., y Barcellos, V. (2006). Artificial Intelligence and Knowledge Management. IFIP International Conference on Artificial Intelligence in Theory and Practice (pp. 11-19). Springer. https://doi.org/10.1007/978-0-387-34747-9_2.

Johannessen, J., Olsen, B. y Olaisen, J. (1999). Aspects of Innovation Theory Based on Knowledge-Management. International Journal of Information Management, 19(2), 121-139. https://doi.org/10.1016/S0268-4012(99)00004-3

Kaplan, A. M., y Haenlein, M. (2010). Users of the World, Unite! The Challenges and Opportunities of Social Media. Business Horizons, 53(1), 59-68.

Nilsson, M., Griggs D., y Visbeck, M. (2016). Policy: Map the Interactions Between Sustainable Development Goals. Nature, 534(7607), 320-322. https://doi.org/10. $1038 / 534320 \mathrm{a}$

Zhenmin, L. (2020). Informe de los objetivos sostenibles 2020. https://unstats.un.org/sdgs/ report/2020/The-Sustainable-Development-Goals-Report-2020_Spanish.pdf 